\title{
Sneak Circuits in Power Converters: Concept, Principle and Application
}

\author{
Bo Zhang and Dongyuan Qiu
}

\begin{abstract}
Sneak Circuit" is defined as the unexpected path or operational status in an electric or electronic circuit due to the limitation or oversight in design by human. The sneak circuit can be triggered to operate under certain conditions, which results in an unwanted or unintended action. As power converter is an artificially designed system, it is undoubted that sneak circuits exist in power converters, while sneak circuit phenomena have been proved in some typical power converters. In order to improve safety and reliability of a power electronic system, it is necessary to understand thoroughly the sneak circuit in power converters under all possible practical conditions. Thus, this paper aims at providing a brief review of sneak circuits in power converters, and summarizes the sneak circuit analysis (SCA) methods based on the graph theory. Finally, some applications on power converters by making use of the SCA principle of sneak circuit analysis are included.
\end{abstract}

Index Terms-Sneak circuit, power converter, graph theory, sneak circuit analysis (SCA).

\section{INTRODUCTION}

$\mathrm{P}$ OWER converter is an important part in most of electrical and electromechanical systems, because it can realize energy transfer between different electrical forms and meet the requirement of high efficiency [1], [2]. As the need of power converters grow rapidly in many areas, the reliability of power converter should be concerned according to its fundamental place in energy conversion and management.

Normally, preventing part failure or component failure is an effective method to improve system reliability [3]. However, not all systems failures are caused by component failure. In some situations, no part has failed, yet the system performs improperly or initiates an undesired function. A significant cause of such unintended events is named as "sneak circuit", which is the unexpected electrical path or logic flow that can produce an undesired result under certain conditions [4]. Opposing to the component failure, sneak circuit happens without any physical failure in the system, an undesired effect is obtained although all parts are working within design specifications.

It has been concluded that the main factor causing sneak circuit is lacking of a complete view of the detailed interrelationship between components and functions in a system [5]. Similar to the other systems, it has been proven that

\footnotetext{
Manuscript received March 27, 2017.

The authors are with School of Electric Power, South China University of Technology, Guangzhou, China (e-mail: epbzhang@scut.edu.cn).

Digital Object Identifier 10.24295/CPSSTPEA.2017.00008
}

sneak circuits exist in the power converter, which may affect the performance of the whole system [6]-[8]. Therefore, the sneak circuit situations in power converters should be investigated and identified, which will have a positive impact on the reliability of power electronic system.

In order to identify sneak circuits systematically, sneak circuit analysis (SCA) is a safety analytical technique which was developed firstly by Boeing in the late 1960s [9]. However, different from other electric or electronic systems, power converter is a typical switched-mode system which operates based on the alternate switching of the semiconductor switches. Obviously, sneak circuit conditions of power converters will relate not only to the variation of parameters, such as the step change of input voltage or load resistance, but also to the control schemes applied for the power switches. Thus, the conventional SCA methods are not suitable for analyzing the sneak circuits in power converters, SCA for power converter should consider both circuit paths and control schemes.

The remainder of this paper is organized in the following sections. Section II gives a comprehensive description of sneak circuit in power converter by using a common Boost DC-DC converter as an example. Section III reviews several available SCA methods for power converters. And then the applications of SCA methods on sneak circuit elimination, topology design and performance improvement are provided in Section IV. Finally, some concluding remarks in the sneak circuit of power converter are drawn in Section $\mathrm{V}$.

\section{Concept of Sneak Circuit}

Sneak circuit is a designed-in current path or signal flow within a system which inhibits wanted functions or causes unwanted functions to occur without a component having failed. Sneak circuits are not the result of component failures, electrostatic, electromagnetic or leakage factors, marginal parametric factors or slightly out-of-tolerance conditions, they are present but not always active conditions inadvertently designed into the system, coded into the software program, or triggered by human error [5]. Consequently, the sneak circuit phenomena are controllable or reversible if the condition of sneak circuit occurrence are known.

\section{A. Sneak circuit definition in power converter}

Power converter is usually composed of power diodes, controllable power switches, inductors, capacitors and resistors. As inductor and capacitor belong to the energy storage components, some unintended electrical current paths can 
be established by inductor and/or capacitor independently. If this kind of current path affects the performance of power converter, they can be considered as the sneak circuit path in power converter, according to the definition of sneak circuit.

In fact, sneak circuit in power converters is not strange. For example, the basic Boost converter shown in Fig. 1(a) has two distinct operating modes based on the status of inductor current, one is continuous conduction mode (CCM) and the other is discontinuous conduction mode (DCM). Generally, Boost converter is designed to operate in CCM or DCM to meet certain control functions by selecting the converter parameters (for example the inductance) in advance. However, CCM will switch to DCM and vice versa, when some operating conditions change, such as input voltage, load or duty ratio. Comparing Fig. 1(b) with Fig. 1(c), if CCM is regarded as the normal operation mode, then the current path only consisting of the output capacitor and the load when both D and S are OFF in DCM can be considered as the sneak circuit path. It is well known that the voltage gain in DCM is different from that in CCM, thus DCM can be considered as the sneak circuit mode.

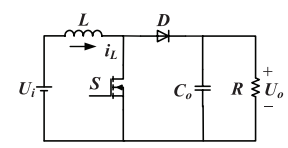

(a) Topology

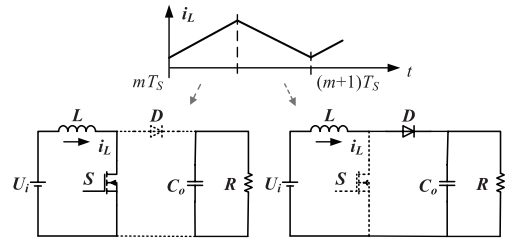

(b) $\mathrm{CCM}$

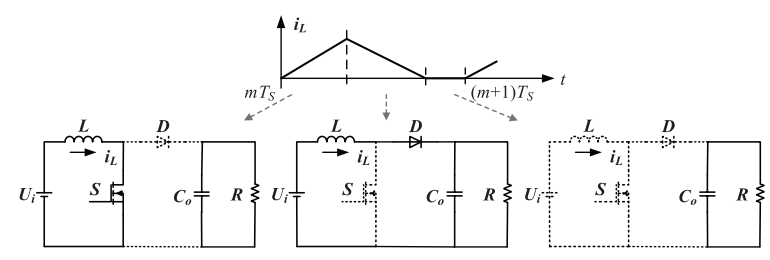

(c) DCM

Fig. 1. Boost converter and its operating modes.

\section{B. Sneak circuit considering parasitic parameter}

The components in power converter are always considered as the ideal ones in order to simplify the analysis, however, all components in power converter have parasitic parameters in fact. It is obviously that the current flows in power converter will be more complicated when all parasitic components are considered, and the probability of overlooking potentially undesirable conditions will increase proportionately as a result.

Take Boost converter as the example again, if Power MOSFET is selected as the power switch $S$, the equivalent circuit of Boost converter is shown in Fig. 2(a) when some critical par- asitic elements are taken into account [10]. The actual model of Power MOSFET is composed of a N-channel MOSFET, a gate-drain capacitor $C_{g d}$, a gate-source capacitor $C_{g s}$, a drainsource capacitor $C_{d s}$, and a body diode $D_{S}$. And power diode $D$ is represented by a freewheel diode in paralleled with a junction capacitor $C_{D}$.

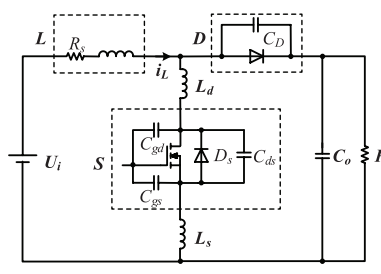

(a) Equivalent circuit

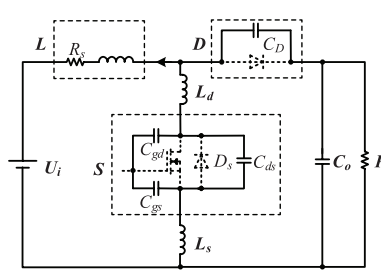

(c) Current path \#2

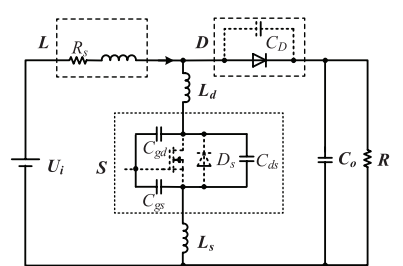

(b) Current path \#1

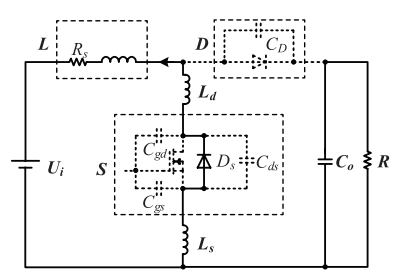

(d) Current path \#3
Fig. 2. Boost converter with parasitic parameters.

When Boost converter operates in DCM, except for the conventional current paths in Fig.1 (c), it is found that there exist another three current paths caused by the parasitic components, which are illustrated in Fig.2 (b) to Fig.2 (d), respectively. The experimental waveforms of a Boost prototype shown in Fig. 3 are provided to prove the existence of the above three current paths [10]. In Case I or Fig. 3(a), current paths \#1, \#2 and \#3 appear, because the voltage on the drain-source capacitor resonates, the inductor current $i_{L}$ is negative and the body diode current $i_{D s}$ is not equal to zero during the switch is turned off. However, $i_{D s}$ keeps at zero in Fig. 3(b), which means that current path \#3 disappears in Case II. By changing some operating conditions of the prototype, the ideal DCM waveform similar to Fig. 1(c) is obtained in Fig. 3(c), it can be concluded that current paths \#1, \#2 and \#3 have not been established in Case III. Therefore, the appearance of these three paths are controllable, there will be currents flowing through these paths during the operating process of Boost converter if certain operating conditions are satisfied. In addition, the influence of different cases as well as each parasitic component on the voltage gain have been summarized in literature [10]. It is demon-

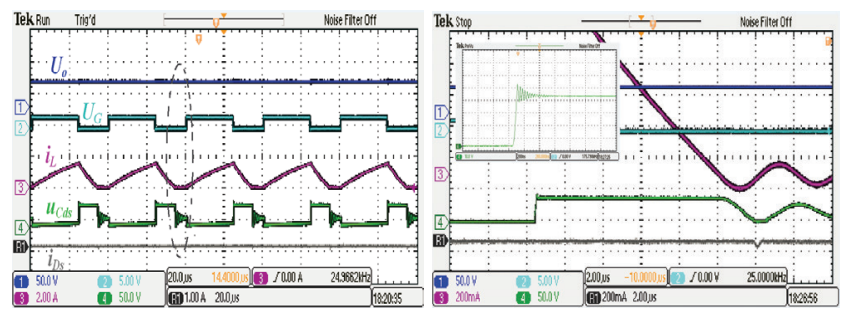

(a) Case I 


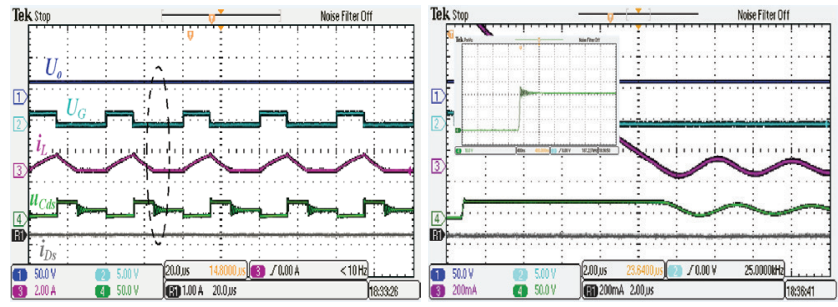

(b) Case II

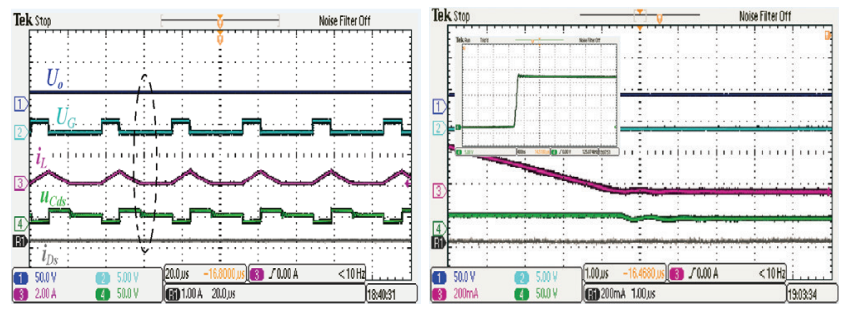

(c) Case III

Fig. 3. Experimental waveforms of Boost converter under different conditions.

strated that current paths caused by parasitic parameters in power converter are coincident with the definition of sneak circuit.

\section{Principle of Sneak Circuit Analysis}

As sneak circuit have been found and proven in a variety of power electronic converters [11], it is necessary to understand thoroughly the sneak circuits in power converter under all possible practical conditions. According to the definition of sneak circuit in power converter, sneak circuit phenomenon belongs to one of the operating modes of power converter. It is known that the operating mode of power converters consists of several operating stages in one switching period, different operating stages are switched in a fixed order according to the applied control strategy. Since each operating stage refers to a sub-circuit or equivalent circuit, there is current flowing through the sneak circuit path when sneak circuit phenomenon appears. Therefore, the sneak circuit analysis of power converter should have two functions: one is sneak circuit path analysis, which is used to identify the sneak circuits paths existed in the converter; the other is sneak circuit mode analysis, which can predict the sneak circuit phenomena.

\section{A. Sneak Circuit Path Analysis}

The precondition to find out the sneak circuit path is to obtain all possible circuit paths or current loops existed in the power converter. If a current path is neither an invalid current path nor a normal current path, then it belongs to the sneak circuit one. Obviously, searching for circuit paths in power converter is the first step in sneak circuit path analysis. If power converter is considered as a graph, some concepts in graph theory, such as adjacency matrix, connection matrix, and mesh, will be useful for path or loop searching [12]-[14].

When a directed graph $G=(V, E)$ with two finite sets $V$ and $E$ is used to describe the power converter, the element of vertex set $V$ represents the intersection of components in the power converter, which is named by number; the element of edge set $E$ is the branch in the converter, which is named by the component symbol of the branch; and the direction of the edge is determined by the current direction of the component on the edge. The edge whose current can flow in bi-direction is indicated by a bidirectional or two-way arrow, while the edge with diode or the switching component without inverse conduction property is indicated by a single-way arrow, which points to the possible flowing direction of the component current.

Boost converter is taken as an example again to explain how to establish the directed graph of power converter. As shown in Fig. 4(a), the branch in the converter is defined as edge, the intersection of the branches is defined as vertex and there are 4 vertices in Boost converter. Considering the symbol of the component on the branch as the edge name, the component current direction as the edge direction, the directed graph of Boost converter can be obtained in Fig. 4(b).

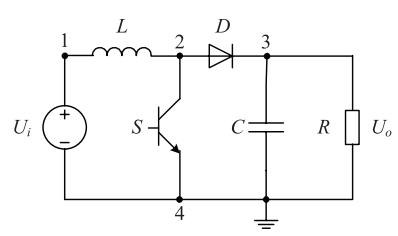

(a) Vertex definition

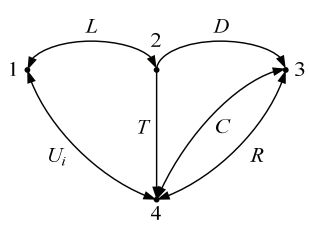

(b) Directed graph
Fig. 4. Boost converter and its directed graph.

According to the definition of adjacency matrix [12], the adjacency matrix of Boost converter is

$$
\mathbf{A}=\left[\begin{array}{llll}
0 & 1 & 0 & 1 \\
1 & 0 & 1 & 1 \\
0 & 0 & 0 & 1 \\
1 & 0 & 1 & 0
\end{array}\right]
$$

Based on (1), the vertex sequence 1-2-3-4 is on behalf of one current path in the Boost converter, because $a_{12}=1, a_{23}=1$, $a_{34}=1, a_{41}=1$. But the vertex sequence 4-3-2-1 is an invalid current path, because $a_{32}=0$. Therefore, all the current paths in power converter can be described by a group of vertex sequences and obtained by some searching algorithms [11].

If the matrix element of the connection matrix is defined by

$$
c_{i j}=\left\{\begin{array}{l}
1, \text { for } i=j \\
0, \text { for } i \neq j \text { and no current path from } i \text { to } j \\
\text { Element symbol, for } i \neq j
\end{array}\right.
$$

then the generalized connection matrix of Boost converter is 


$$
\mathbf{C}=\left[\begin{array}{cccc}
1 & L & 0 & U_{i} \\
L & 1 & D & S \\
0 & 0 & 1 & R+C_{o} \\
U_{i} & 0 & R+C_{o} & 1
\end{array}\right]
$$

As the current path must be a loop in the circuit, all possible current loops in power converter can be found by calculating the determinant of the generalized connection matrix $\mathbf{C}$ [15], [16]. Thus, base on (3), the calculation result of loop is

$$
f_{\text {loop }}=|\operatorname{det} \mathbf{C}| \Rightarrow R C_{o}+U_{i} L D R+U_{i} L D C_{o}+U_{i} L S
$$

From (4), it is concluded that there are 4 current loops in Boost converter, which are $R-C_{o}, U_{i}-L-D-R, U_{i}-L-D-C_{o}$ and $U_{i}-L-S$, respectively.

Since an operating stage of power converter is normally consisted of more than one circuit path or current loop, circuit paths or current loops should be combined to a sub-circuit which refers to an operating stage. By defining mesh as the loop that does not contain internal loop with edge [14], mesh combination algorithm can be used to find out all of the possible sub-circuits in power converter.

According to the mesh definition, Boost converter in Fig. 1(a) is made up of 3 meshes, which are expressed by the following sets:

$$
\begin{aligned}
& G_{1}=\left\{U_{i}, L, S\right\} \\
& G_{2}=\left\{S, D, C_{o}\right\} \\
& G_{3}=\left\{C_{o}, R\right\}
\end{aligned}
$$

By applying AND $(\cup)$ and RING SUM $(\oplus)$ operations of graph, meshes can be composed into connected pieces [14], for example,

$$
\begin{gathered}
G_{1} \cup G_{3}=\left\{U_{i}, L, S, C_{o}, R\right\} \\
G_{1} \oplus G_{3}=\left\{U_{i}, L, S, C_{o}, R\right\} \\
G_{1} \cup G_{2} \oplus G_{3}=\left\{U_{i}, L, D, C_{o}, R\right\}
\end{gathered}
$$

It can be found that $\mathrm{G} 1(\cup$ or $\oplus) \mathrm{G} 3, \mathrm{G} 1 \cup \mathrm{G} 2 \oplus \mathrm{G} 3$ and $\mathrm{G} 3$ represent for three operating stages in DCM shown in Fig. 1 (c), respectively.

Thus, all possible circuit paths, circuit loops and sub-circuits in power converter can be obtained by the above methods, and they can be divided into groups according to the circuit principle and the operating principle of power converter: 1) The invalid path, loop or sub-circuit, which will not be allowed in the power converter. For example, both $S$ and $D$ are "ON" at the same time in Boost converter. After eliminating the invalid path, the rest of possible paths are the effective ones.

2) The desired path, loop or sub-circuit, which will show up as designed during normal operation of the converter.

3) The sneak circuit path, which is the effective circuit path different from any normal circuit path.
As a result, the sneak circuit path can be identified by comparing the effective circuit paths with the normal ones.

\section{B. Sneak Circuit Mode Analysis}

Even the sneak circuit paths have been obtained, the behavior of the converter when the sneak circuit paths appear during the operating process is still unknown, thus sneak circuit mode analysis should be carried out. It is known that the switching components in the power converter are turned on or off in a fixed order based on the control strategy, so the sub-circuits corresponding to different switching states will constitute the operating mode of power converter.

As shown in Fig. 5, there are two switching components in Cúk converter, one is the controllable switch $S$ and the other is the uncontrollable diode $D$. If " 1 " indicates the state of the switching component when it is on, and " 0 " indicates off, then the available switching states for Cúk converter are expressed by $\mathrm{SD}=10, \mathrm{SD}=01$ and $\mathrm{SD}=00$, while $\mathrm{SD}=11$ is invalid. These three switching states can compose two switch control sequences, 10-01-10-01-... (Control sequence I) and 10-01-00-10-01-00-... (Control sequence II).

The equivalent circuits corresponding to switch states $\mathrm{SD}=$ 10 and $\mathrm{SD}=01$ are shown in Figs. 6(a) and (b), respectively. But both circuits in Figs. 6(c) and (d) refer to the same switch state $\mathrm{SD}=00$. Thus, the operating mode under control sequence I is unique, and there are two operating modes under control sequence II. It is common to define the operating mode under control sequence I (i.e. CCM) as the normal operation mode of Cúk converter, while those under control sequence II should be considered as sneak operating modes, which are shown in Figs. 7(a) and (b).

In order to describe the sneak circuit phenomena of Cúk converter, the waveforms of inductor currents $i_{\mathrm{L} 1}$ and $i_{\mathrm{L} 2}$ are selected. It is noted that inductors $L_{1}$ and $L_{2}$ are connected in

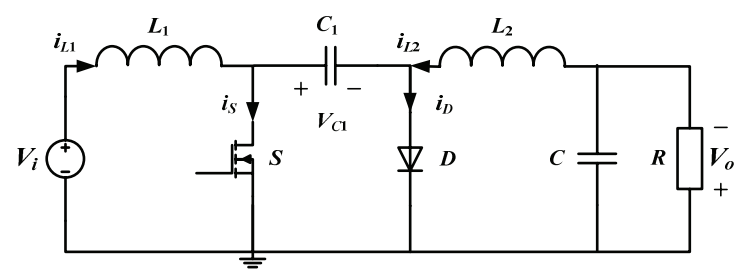

Fig. 5. Schematic of Cúk converter.

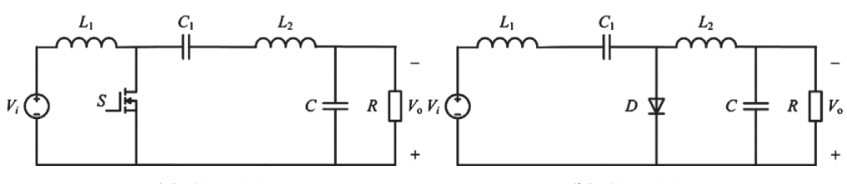

(a) $\mathrm{SD}=10$

(b) $\mathrm{SD}=01$

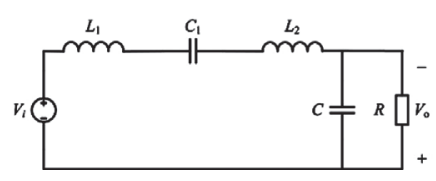

(c) $\mathrm{SD}_{(1)}=00$

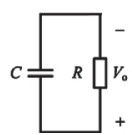

(d) $\mathrm{SD}_{(2)}=00$
Fig. 6. Equivalent circuits of Cúk converter. 


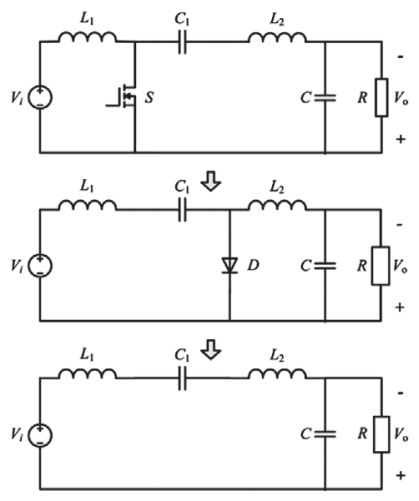

(a) Sneak operating mode 1

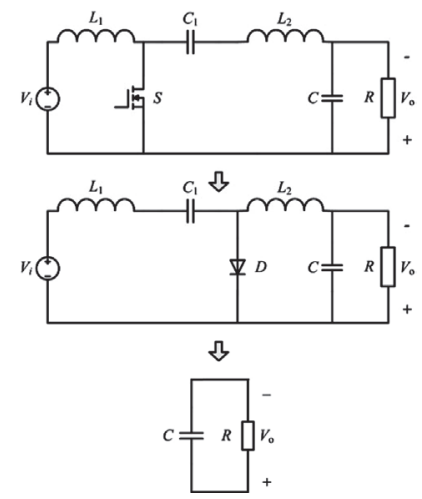

(b) Sneak operating mode 2
Fig. 7. Sneak operating modes of Cúk converter.

series in Fig. 6(c), then $i_{\mathrm{L} 1}+i_{\mathrm{L} 2}=0$ should be satisfied, and the inductor currents will have the following two forms: (i) $i_{\mathrm{L} 1}<0$ and $i_{\mathrm{L} 2}>0$; (ii) $i_{\mathrm{L} 1}>0$ and $i_{\mathrm{L} 2}<0$. When Cúk converter operates in Fig. 6(d), both of the inductor currents $i_{\mathrm{L} 1}$ and $i_{\mathrm{L} 2}$ are equal to zero, i.e. $i_{\mathrm{L} 1}=i_{\mathrm{L} 2}=0$. Therefore, based on the possible current forms of the equivalent circuits, the typical inductor waveforms corresponding to sneak operating modes can be predicted and shown in Fig. 8.

A Cúk prototype has been built to verify different kinds of phenomena [17]. By reducing the duty cycle $d$, the Cúk prototype will work in different operating modes, and the experimental waveforms of driving signal $v_{s}$, output voltage $V_{o}$, inductor currents $i_{\mathrm{L} 1}$ and $i_{\mathrm{L} 2}$ are shown in Fig. 9, which have good agreement with the predicted waveforms in Fig. 8.
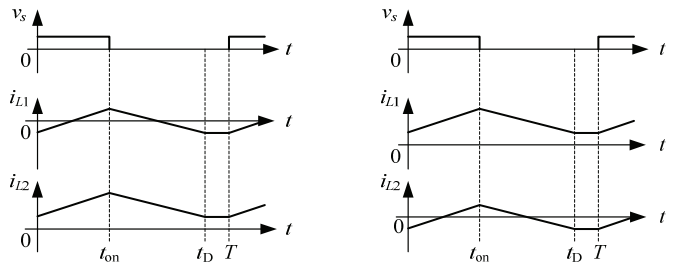

(a) Typical waveforms of sneak operating mode 1

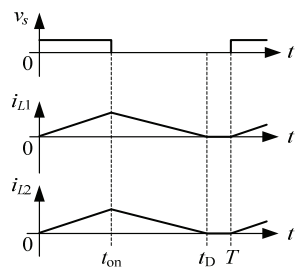

(b) Typical waveforms of sneak operating mode 2

Fig. 8. Sneak circuit phenomena of Cúk converter.

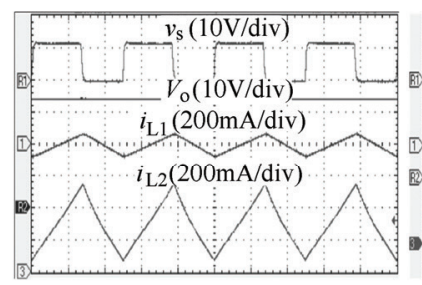

Time $(4 \mu \mathrm{s} / \mathrm{div})$

(a) Normal operating mode (or CCM)

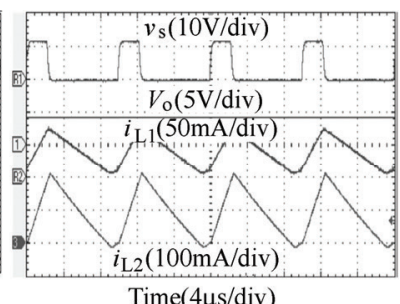

(b) Sneak operating mode 1(ii)

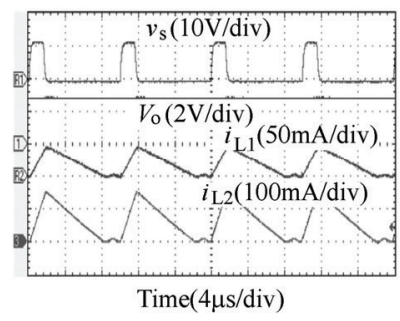

(c) Sneak operating mode 2

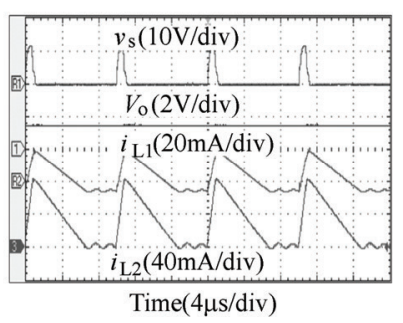

(d) Sneak operating mode 1(i)
Fig. 9. Experimental waveforms of different operating mode.

Therefore, the feasibility of the proposed sneak circuit mode analysis method has been verified.

\section{Application of SneAK Circuits}

With the help of sneak circuit analysis method proposed in section III to power converter, the operating principle and the characteristics of power converter can be understood comprehensively, thus SCA for power converter is useful to modify the topology and improve the performance of the power converter.

\section{A. Sneak Circuit Elimination}

Based on the definition of sneak circuit, the sneak circuit of power converter is a path hided in the converter, which would appear when specific parameter condition is satisfied. As a result, the operating stages increase and the energy balance under normal operating mode is destroyed, which will lead to some changes of converter characteristics and the desired performance of the converter cannot be achieved. Thus the sneak circuits should be eliminated in the power converter.

Apparently, the emergence of sneak circuits should satisfy two conditions: firstly, there must be some sneak circuit paths existed in the power converter; secondly, sneak circuits will appear if the converter parameters are not designed appropriately or the converter operating conditions are out of the normal range. Therefore, in order to eliminate sneak circuits in power converters, there are two methods:

1) Proper parameter design, which is suitable for the converter which has specific sneak circuit condition, because the emergence of sneak circuit can be avoided through reasonable parameter design.

2) Topology modification, which can cut off the sneak circuit paths by changing the converter topology based on the characteristic of sneak circuit path.

The three-phase Z-source inverter [18] shown in Fig. 10 is taken as an example to demonstrate how to eliminate the sneak circuit by parameter design and topology modification. Normally, the diode $D$ conducts when the three-phase inverter is at the active or traditional zero state, $D$ is blocked when the inverter operates at the shoot-through state. However, when the diode current is discontinuous during the period of active states, the input dc voltage of the inverter bridge drops, which will affect the quality of the output ac voltage seriously. It has been proven that this sneak circuit phenom- 
ena will occur if a small inductance or low load power factor is selected [19].

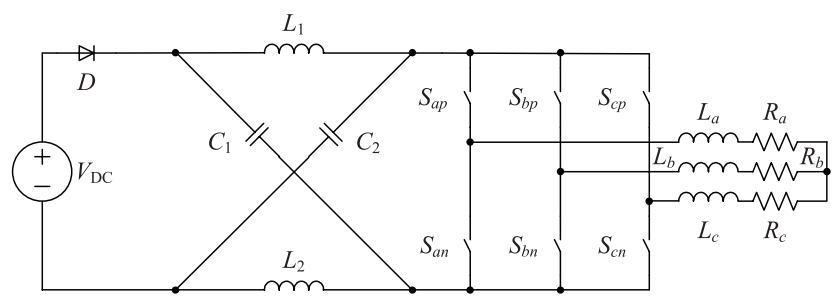

Fig. 10. The classic three phase Z-source inverter.

As the Z-source inverter has a specific sneak circuit condition, if the operating conditions of $Z$-source inverter are set to satisfy with the following equation, the sneak circuit phenomenon of Z-source inverter will not appear.

$$
\frac{3}{2} M B \cos \phi-1 \geq 0
$$

where $M$ is modulation ratio, $B$ is step-up factor and $\cos \phi$ is the load power factor.

On the other hand, the sneak circuit phenomenon will appear in the Z-source inverter when the link between the DC voltage source $V_{\mathrm{DC}}$ and impedance network is open during the inverter's active state. Therefore, if there is a circuit path that connects $V_{\mathrm{DC}}$ with Z-network at any state, the sneak circuit phenomenon will disappear. Substituting diode $D$ by a fully controlled power switch $S_{m}$ which has the reverse conducting property (i.e. Power MOSFET), the improved Z-source inverter is illustrated in Fig. 11.

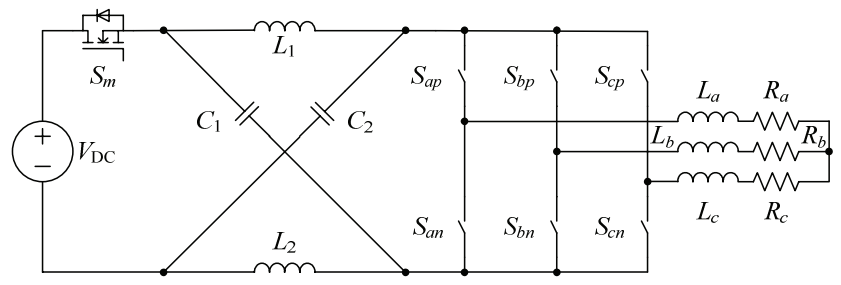

Fig. 11. The improved Z-source inverter.

As the input current or the switch current can flow bi-directionally, the switch can provide a backward path for the current by its control signal or anti-parallel diode even when the input current decreases to zero and becomes reversed. Therefore, the sneak circuit phenomenon of the classic Z-source inverter will not happen forever.

\section{B. Performance Improvement}

In fact, as the existed circuit paths in power converter, sneak circuits may have positive effect in power converter if it can be made good use of. The synchronous Buck converter can be used as an example to discuss how to improve the converter performance without adding any component but by making use of the sneak circuit.
The synchronous Buck converter can be obtained by replacing the diode in Buck converter with a controllable power switch. As shown in Fig. 12, $S_{1}$ is the main switch and $S_{2}$ is the synchronous switch. In general, the conduction of $S_{1}$ and $S_{2}$ are complementary, and certain dead time is inserted between the two driving signals to prevent simultaneous conduction of the two switches.

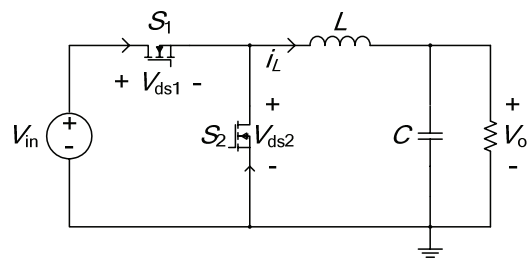

Fig. 12. Synchronous Buck converter.

When the inductor current is continuous and positive, typical waveforms of the synchronous Buck converter in normal operating mode are shown in Fig. 13(a). It is obvious that the synchronous switch $S_{2}$ can achieve ZVS before it is switched on. However, the main switch $S_{1}$ is still operating in hard-switching state. As the switch such as Power MOSFET can flow through bidirectional current, the body diode of $S_{1}$ will conduct when the inductor current $i_{\mathrm{L}}$ drops to zero and becomes negative during the on-state of $S_{2}$, then the zero-voltage turn-on condition of the main switch $S_{1}$ can be created. The typical waveforms that both the main switch and the synchronous switch can achieve ZVS are shown in Fig. 13(b).

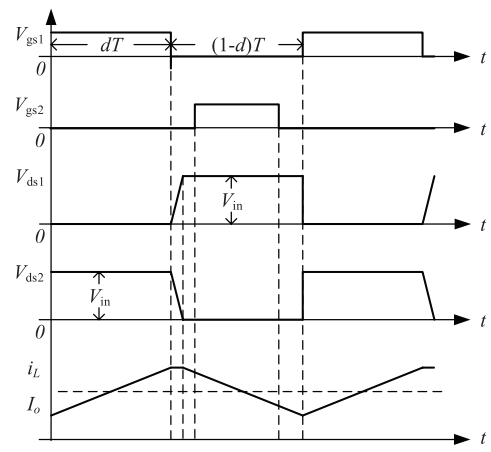

(a) Normal waveforms

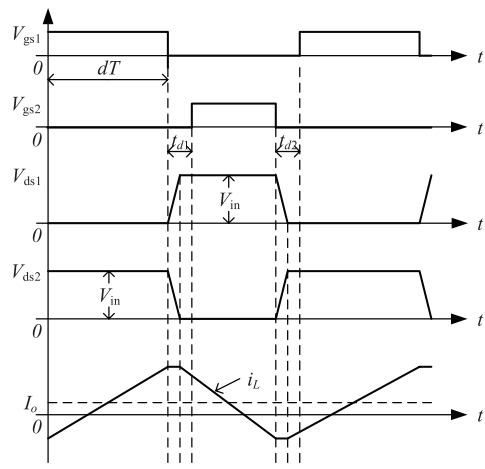

(b) Sneak circuit mode

Fig. 13. Typical waveforms of Synchronous Buck converter under different modes. 
It has been proven that this situation only happen when the inductance of the synchronous Buck converter is designed relatively small [20], thus Fig. 13(b) can be regarded as the sneak circuit phenomenon of the synchronous Buck converter according to the definition of sneak circuit. Obviously, it is a significant reward to achieve ZVS by using the sneak circuit paths that exist in synchronous DC-DC converter.

\section{Topology Reconstruction}

Topology is one of the key techniques in power electronics, because it determines the basic performance and operating characteristics of power converter. A large number of power converter topologies have been proposed, such as Cúk converter [21], resonant converter [22], soft-switching converter [23], multilevel converter [24], modular multilevel converter (MMC) [25], etc., which have greatly improved the level and quality of electric energy transformation.

However, it is found that the invention of the novel power converter largely depends on the inventors' academic attainments and practical experiences. Furthermore, many typical power converters also need further improvement and optimization in practical application and the process of topology reconstruction is still based on the "try and test" method. As the sneak circuit analysis method can provide a thoroughly understanding of power converter, it is possible to be applied in reconstructing the topology of power converter.

For example, the original Boost ZCT PWM converter and an improved Boost ZCT PWM converter are shown in Fig. 14 and Fig. 15, respectively.

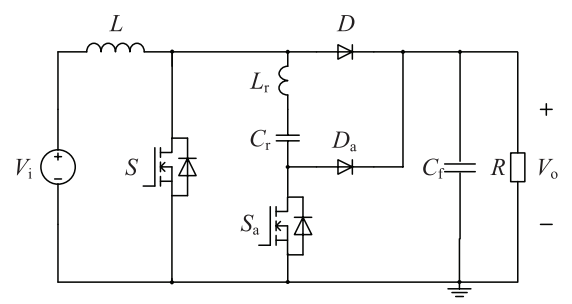

Fig. 14. The original Boost ZCT PWM converter.

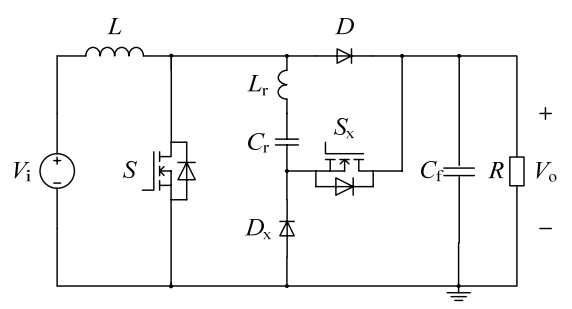

Fig. 15. An improved Boost ZCT PWM converter.

Comparing Fig. 15 with Fig. 14, diode $D_{\mathrm{x}}$ substitutes for the original auxiliary switch $S_{a}$ and the conducting direction of $D_{\mathrm{x}}$ is consistent with the anti-parallel diode of $S_{\mathrm{a}}$; switch $S_{\mathrm{x}}$ substitutes for the original auxiliary diode $D_{\mathrm{a}}$ and the anti-parallel diode of $S_{\mathrm{x}}$ keeps the same conducting direction of $D_{\text {a. }}$. Therefore, the switching states of both converters are the same, the original functions of Boost ZCT PWM converter can be kept in the modified one. Furthermore, the modified Boost ZCT PWM converter is proven to have other advantages, such as ZCS of the main switch as well as auxiliary switch can be realized, the peak current of auxiliary switch is relatively small and the switch time of auxiliary switch is easy to control [26]. Therefore, topology reconstruction method by making use of the sneak circuit path analysis method is feasible, and the good performance of the original converter can be kept.

\section{Conclusions}

In this paper, the work on sneak circuits and related sneak circuit analysis methods for power converters have been reviewed, how to eliminate and make use of sneak circuit have been proposed as well. The research on sneak circuit in power converters offers guidelines in the design of power electronic system and enable early detection of potential problems, which will contribute to the reliability of power electronic system.

\section{REFERENCE}

[1] J. D. van Wyk, and F. C. Lee, "On a future for power electronics," IEEE Journal of Emerging and Selected Topics in Power Electronics, vol.1, no. 2, pp.59-72, 2013.

[2] D. Divan, and P. Kandula, "Distributed power electronics: An enabler for the future grid", CPSS Transactions on Power Electronics and Applications, vol.1, no.1, pp.57-65, 2016

[3] Y. Yang, A. Sangwongwanich, and F. Blaabjerg, "Design for reliability of power electronics for grid-connected photovoltaic systems", CPSS Transactions on Power Electronics and Applications, vol.1, no.1,pp.92-103, 2016.

[4] United States Navy, Sneak circuit analysis: A means of verifying design integrity, University of Michigan Library, 1986.

[5] D. L. Buratti, and S. G., Godoy, "Sneak analysis application guidelines", Rome Air Development Center (RADC) Tech Rep, TR-82179, 1982.

[6] D. Qiu, and B. Zhang, "Analysis of step-down resonant capacitor converter with sneak circuit state," in Proceedings of IEEE Power Electronics Specialists Conference (PESC), 2006, pp.2940-2944.

[7] D. Qiu, and B. Zhang, "Discovery of sneak circuit phenomena in resonant switched capacitor DC-DC converters", in Proceedings of the 1st IEEE Conference on Industrial Electronics and Applications (ICIEA), 2006, pp. 993-996.

[8] W. Tu, D. Qiu, B. Zhang, and J. Li, "General laws of sneak circuit in resonant switched capacitor converters", in Proceedings of 38 th IEEE Power Electronics Specialists Conference (PESC), 2007, pp.708-712.

[9] J. P. Rankin, Sneak-circuit analysis, vol.14, no.5, Nuclear Safety, 1973, pp.461-469.

[10] M. Li, B. Zhang, D. Qiu, and G. Zhang, "Sneak circuit phenomena in a DCM Boost converter considering parasitic parameters", IEEE Transactions on Power Electronics, vol. 32, no.5, pp.3946-3958, 2017.

[11] B. Zhang, and D. Qiu, Sneak Circuits of Power Electronic Converters, Singapore: Wiley-IEEE, 2014

[12] R. Diestel, Graph Theory, New York, USA: Springer-Verlag, 2000.

[13] J. A. Bondy and U. S. R. Murty, Graph theory with Applications. North-Holland, The Netherlands: Elsevier Science Ltd., 1976.

[14] W. K. Chen, Applied Graph Theory: Graphs and Electrical Networks. 2nd ed. Amsterdam, The Netherlands: North- Holland Publishing Co., 1976.

[15] J. Li, D. Qiu, and B. Zhang, "Sneak circuit analysis for n-stage resonant switched capacitor converters based on graph theory", in 
Proceedings of the 33rd Annual Conference of the IEEE Industrial Electronics Society (IECON), 2007, pp.1581-1585.

[16] L. Qu, B. Zhang, D. Qiu, and W. Tu, "Sneak circuit analysis method based on generalized connection matrix for power converters", in Proceedings of International Conference on Electrical Machines and Systems (ICEMS), 2008, pp. 1587-1590.

[17] B. Zhang, D. Qiu, and G. Yi, "Multiple operating mode analysis of power converter based on graph theory", Chinese Journal of Electrical Engineering, vol.1, no.1, pp.70-77, 2015.

[18] F. Z. Peng, "Z-source inverter," IEEE Transactions on Industry Applications, vol. 39, no.2, pp. 504-510, 2003

[19] M. Shen, and F. Z. Peng, "Operation modes and characteristics of the Z-source inverter with small inductance or low power factor", IEEE Transactions on Industry Electronics, vol.55, no.1, pp.89-96, 2008.

[20] C. P. Henze, H. C. Martin, and D. W. Parsley, "Zero-voltage switching in high frequency power electronic converters using pulse width modulation", in Proceedings of IEEE Applied Power Electronics Conference and Exposition (APEC), 1988, pp.33-40.

[21] S. Cúk, and R. D. Middlebrook, "A new optimum topology switching DC-to-DC converter," in Proceedings of IEEE Power Electronics Specialists Conference (PESC), 1977, pp.160-179.

[22] K. Liu, and F. C. Lee, "Resonant switches-a unified approach to improve performances of switching converters," in Proceeding of IEEE International Telecommunications Energy Conference, 1984, pp.334-341.

[23] K. Liu, and F. C. Lee, "Zero-voltage switching technique in DC/DC converters," IEEE Transaction on Power Electronics, vol.5, no. 3, pp.293-304, 1990.

[24] A. Nabae, I. Takahashi, and H. Akagi, "A new neutral-point-clamped PWM inverter," IEEE Transaction on Industry Applications, vol. IA17, no.5, pp.518-523,1981.

[25] A. Lesnicar, and R. Marquardt, "An innovative modular multilevel converter topology suitable for a wide power range," in Proceeding of IEEE Powertech Confrenenle., 2003, pp.1-6.
[26] H. Mao, F. C. Y. Lee, X. Zhou, et al., "Improved zero-current transition converters for high-power applications", IEEE Transactions on Industry Applications, vol.33, no.5, pp.1220-1232, 1997.

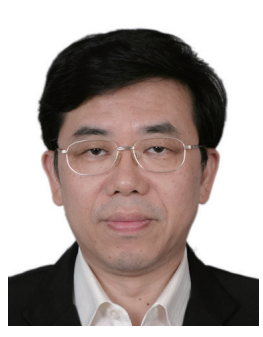

Bo Zhang received the B.S. degree in electrical engineering at Zhejiang University, Hanzhou, China, in 1982, the M.S. degree in power electronics at Southwest Jiaotong University, Chengdu, China, in 1988, and the Ph.D. degree in power electronics at Nanjing University of Aeronautics and Astronautics, Nanjing, China, in 1994.

$\mathrm{He}$ is currently a Professor in the School of Electric Power, South China University of Technology, Guangzhou, China. He has authored or coauthored 2 books in Wiley, more than 400 papers and 110 patents. His current research interests include nonlinear analysis and control of power electronic systems, wireless power transfer and ac drives.

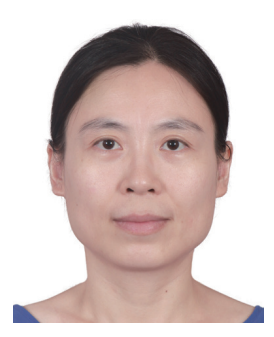

Dongyuan Qiu received the B.Sc. and M.Sc. degrees in Automation at South China University of Technology, Guangzhou, China, in 1994 and 1997, respectively, and the Ph.D. degree in Power Electronics at City University of Hong Kong, Kowloon, Hong Kong, in 2002.

She is currently a Professor in the School of Electric Power, South China University of Technology. Her main research interests include sneak circuit analysis and fault diagnosis of power electronic systems, design and control of power converters. She has published one book and over 100 journal papers in these areas. 